\title{
Automatic Detection and Recognition of Tonal Bird Sounds in Noisy Environments
}

\author{
Peter Jančovič (EURASIP Member) and Münevver Köküier \\ School of Electronic, Electrical \& Computer Engineering, University of Birmingham, Birmingham, B15 2TT, UK \\ Correspondence should be addressed to Peter Jančovič, p.jancovic@bham.ac.uk
}

Received 13 September 2010; Revised 24 December 2010; Accepted 7 February 2011

Academic Editor: Tan Lee

Copyright () 2011 P. Jančovič and M. Köküer. This is an open access article distributed under the Creative Commons Attribution License, which permits unrestricted use, distribution, and reproduction in any medium, provided the original work is properly cited.

\begin{abstract}
This paper presents a study of automatic detection and recognition of tonal bird sounds in noisy environments. The detection of spectro-temporal regions containing bird tonal vocalisations is based on exploiting the spectral shape to identify sinusoidal components in the short-time spectrum. The detection method provides tonal-based feature representation that is employed for automatic bird recognition. The recognition system uses Gaussian mixture models to model 165 different bird syllables, produced by 95 bird species. Standard models, as well as models compensating for the effect of the noise, are employed. Experiments are performed on bird sound recordings corrupted by White noise and real-world environmental noise. The proposed detection method shows high detection accuracy of bird tonal components. The employed tonal-based features show significant recognition accuracy improvements over the Mel-frequency cepstral coefficients, in both standard and noise-compensated models, and strong robustness to mismatch between the training and testing conditions.
\end{abstract}

\section{Introduction}

Identification of birds, the study of their behavior, and the way of their communication is important for a better understanding of the environment we are living in and in the context of environmental protection. Bird species identification currently relies crucially on expert ornithologists who identify birds by sight and, more often, by their songs and calls. In recent years, there has been an increased interest in automatic recognition of bird species using the acoustic signal.

Bird vocalisation is usually considered to be composed of calls and songs, which consist of a single syllable or a series of syllables. Sounds produced by birds may be of a various character. Some birds produce sounds of a noisy broadband character, but most produce a tonal sound, which may consist of a pure tone frequency, several harmonics of the fundamental frequency, or several non-harmonically related frequencies [1]. The bird sounds are often modulated in both frequency and amplitude. Field recordings of bird vocalisations in their natural habitat are usually contaminated by various noise backgrounds or vocalisations of other birds or animals.
Automatic recognition of bird species based on their sounds is a pattern recognition problem, and as such, it consists of a feature extraction stage that aims to extract relevant features from the signal and a modelling stage that aims to model the distribution of the features in space. Early attempts at automatic bird recognition were based on template matching of signal spectrograms using dynamic time warping (DTW), for example, see [2]. The study in [2] was performed on two birds and involved manual segmentation of the templates of representative syllables. The authors in [3] compared the use of DTW and hidden Markov models (HMMs) on recognition of bird song elements from continuous recordings of two bird species. Artificial neural networks (NNs) have also been applied to the recognition of bird sounds; for example, see [4-6]. The backpropagation neural network was used in [4], a combined time delay NNs with an autoregressive version of the backpropagation in [5], and a recurrent neural fuzzy network in [6]. Recently, Gaussian mixture models (GMMs) have also been used for recognition of bird sounds; for example, see $[7,8]$. These studies also compared the recognition performance obtained by employing the GMMs and HMMs 
and reported only small differences in performance. The use of support vector machines was presented in [9] and neural network classifiers employing wavelets in [10], however, neither works presented any comparison to GMMs or HMMs.

Various feature representations of bird sounds for automatic bird recognition have been explored. Many of the studies were inspired by feature representations used in the automatic speech recognition field. Filter-bank energies were used in [3], linear prediction cepstral coefficients in $[4,5]$, and Mel-frequency cepstral coefficients (MFCC) in $[3,7-9,11]$. Features relating to a dominant energy region in the spectrum were used in [12]. The authors in [8] compared three different representations: MFCC features, features based on sinusoidal modelling presented in [13] which estimates sinusoidal components present in the signal, and a set of low-level descriptive features. They reported that MFCC features obtained the best performance. In [9], the combination of MFCC features with a set of low-level signal parameters was shown to slightly improve the recognition performance.

The above-mentioned bird recognition studies performed the recognition using a relatively small number of bird species (between two to sixteen), and nearly all studies were performed on clean data. In [14], it was mentioned that part of the data, which was also used in $[8,9]$, was obtained from field recordings containing some background noise. However, there was no formal evaluation of the noise level and dealing with the background noise was not the concern of their work. The aim of our study in this paper is to investigate automatic detection and recognition of bird sounds in noisy environments. We focus on tonal bird sounds as many of the bird sounds are of a tonal character. The detection of spectro-temporal regions of tonal bird sounds is performed by a method exploiting the spectral shape to identify sinusoidal components in the short-time spectrum. We have introduced this method earlier for voicing character estimation of speech signals [15] and employed it for automatic speech and speaker recognition $[16,17]$ and speech alignment [18]. Here, we will explore the employment of this method for bird acoustic signals. The experimental evaluations are performed on bird data from [19], which is corrupted by White noise and real-world waterfall noise [20] at various signal-to-noise ratios (SNRs). The proposed detection method when used at a frame-level shows that over $95 \%$ of the bird signal frames can be detected as tonal while keeping the false detection on White noise at only $1 \%$. Motivated by the detection method, we then study the feature representation for automatic recognition of bird syllables in noisy conditions. The recognition task consists of 165 different bird syllables produced by 95 bird species. The modelling of the bird sounds is performed by employing Gaussian mixture models. The performance achieved by using the tonal-based feature representation obtained by the proposed detection method is compared with MFCC features. The experimental evaluations are performed using a standard model that is trained on clean data and also using a model that compensates for the effect of the noise. The multi-condition training approach is used for the latter. Experimental results show that both the MFCC features and the tonal-based features can obtain a very high recognition performance in clean conditions. In noisy conditions, the tonal-based features achieve a significantly better performance than the MFCC features in both the standard model and the noise-compensated model. Moreover, the tonal-based features show strong robustness to a mismatch between the training and testing conditions, while the performance of the MFCC features deteriorates significantly even at high SNRs.

The rest of this paper is organised as follows: Section 2 presents the proposed method for the detection of tonal spectro-temporal regions and its evaluation at a frame and spectral-level; Section 3 presents the employment of the tonal-based features for bird recognition employing the Gaussian mixture modelling with experimental evaluations on standard and noise-compensated models; Section 4 presents the discussion and conclusions.

\section{Detection of Bird Sounds in Noise}

This section presents a method for the detection of tonal regions of bird sounds at the spectral-level and framelevel. The method is based on the detection of sinusoidal components in the spectrum based on the spectral shape.

2.1. Principle. As a result of short-time processing, the short-time Fourier spectrum of a sinusoidal signal is the Fourier transform of the frame-window function. Thus, the detection of bird spectral components of a tonal character can be performed based on comparing the short-time magnitude spectrum of the signal to the spectrum of the frame-window function [15].

2.2. Method Description. The steps of the method used for the detection of the bird tonal components in the spectrum are as follows.

(1) Short-Time Magnitude Spectrum Calculation. A frame of a time-domain signal is multiplied by a frame-window function. The Hamming window was employed as a window function due to its good tradeoff between the main-lobe width and side-lobe magnitudes. It was experimentally demonstrated in [15] that the Hamming window provided better detection performance than the rectangular and Blackman-Harris windows (as examples of a narrower and wider main-lobe width, resp.) on simulated sinusoidal signals. In order to obtain a smoother short-time spectrum, the windowed signal frame was appended with zeros, resulting in a signal frame of twice as long as the original signal frame, and the FFT was then applied to provide the short-time magnitude spectrum.

(2) Sine-Distance Calculation. For a frequency point $k$ of the short-time magnitude spectrum, a distance, referred to as sine-distance and denoted by $s d(k)$, between the signal 
spectrum around the point and magnitude spectrum of the frame-window function is computed as

$$
s d(k)=\left[\frac{1}{2 M+1} \sum_{m=-M}^{M}\left(\frac{|S(k+m)|}{|S(k)|}-\frac{|W(m)|}{|W(0)|}\right)^{2}\right]^{1 / 2},
$$

where $M$ determines the number of points of the spectrum at each side around the point $k$ to be compared, and this was set to 3. In (1), the magnitude spectrum of the signal, $S(k)$, and frame window, $W(k)$, are normalised as to have the value equal to 1 when $m=0$. This ensures that the magnitude difference is eliminated and only the shape is being compared. The value of the sine-distance in (1) will be low, ideally equal to zero, when the frequency point $k$ corresponds to a sinusoidal component in the signal; otherwise, it will be high. The sine-distance $s d(k)$ can be calculated for each frequency point in the spectrum or for spectral peaks only. In the latter case, the peaks can be identified by detecting changes of the slope of $S(k)$ from positive to negative.

(3) Postprocessing of the Sine-Distances. The sine-distance obtained from (1) may accidentally be of a low value for a non-tonal region or vice versa. This can be improved by filtering the obtained sine-distances. We employed a $2 \mathrm{D}$ median filter of size $15 \times 3$, where the first and second dimension sizes correspond to the number of frames and spectral points, respectively.

An example of a waveform and spectrogram of a clean tonal bird sound and corrupted by White noise at the global SNR of $-10 \mathrm{~dB}$ and the corresponding sine-distance values are depicted in Figure 1. The frame length and frame shift used here were 64 and 32 samples, respectively. We can see from the spectrogram that the singing frequency of the bird often changed quickly. For instance, in the first segment (within the first $100 \mathrm{~ms}$ ), the frequency changed from $8950 \mathrm{~Hz}$ to $5850 \mathrm{~Hz}$ during approximately $20 \mathrm{~ms}$. Despite these fast frequency variations, the sine-distance shows good detection, that is, low values well tracking the bird singing frequency. For the noise-corrupted bird sound, we can see that while the signal is strongly corrupted by noise, the sinedistance values show a clear detection of the correct bird tonal regions.

\subsection{Experimental Evaluation of Tonal Bird Detection}

2.3.1. Database Description. The experimental evaluations presented throughout this paper were performed using bird data from commercially available bird recordings in [19], which contains the songs and calls of birds living in eastern and central North America on three CDs. The entire collection of bird recordings from the third CD was used. It contains recordings of 99 different types of birds with various character of sounds, ranging from tonal sounds that contain a single frequency, several harmonics, or several nonharmonically related frequencies to some non-tonal sounds and from relatively stationary to highly transient. The signals are recorded at a $44100 \mathrm{~Hz}$ sampling frequency with 16 bits for each sample. The noisy bird data was created by artificially adding noise to the original data at global SNRs of $10 \mathrm{~dB}, 0 \mathrm{~dB}$ and $-10 \mathrm{~dB}$, respectively. As noise source, White noise is used in the experimental evaluations in this section.

2.3.2. Experimental Results. First, we present experimental evaluations of the detection of tonal bird signal frames in clean and noisy conditions. To account for the fact that bird sounds may consist of a single frequency component, a signal frame is considered as tonal if at least one spectral point was detected as tonal. Since the bird database contains bird sounds of various character, and there is no label information indicating which part of the signal is of a tonal character, we adopted the following evaluation methodology. The ideal detector would be expected to detect all the tonal frames in the bird data and at the same time not to detect any frames on White noise as this noise does not contain any pure tonal components. Thus, the evaluation of the detection performance is presented in terms of the percentage of frames detected as tonal on bird data (clean and noisy) versus the percentage of frames detected as tonal on White noise and the latter is referred to as false-acceptance error. Since birds often vary the singing frequency over a short time period, it is important to assess the effect of the frame length on the detection performance. A shorter length of the frame may provide less variations of the signal within the frame, however, it also reduces the frequency resolution of the spectrum.

The experimental results of the detection on clean and noisy data at various global SNRs when using various frame lengths are presented in Figure 2. Note that the individual results presented in the figures correspond to a specific value of the tonal-threshold used, and as the value of the tonalthreshold increases, the false-acceptance increases.

Let us first analyse the results on clean data. We can see that at a given false-acceptance error, the frame length of 32 samples provides the highest percentage of bird frames detected as tonal on the clean data. For instance, at a $2 \%$ false-acceptance error around $96 \%$ of all the signal frames are detected as tonal when the frame length is 32 samples, while the detection drops to around $92 \%$ and $73 \%$ for the frame length of 64 samples and 128 samples, respectively. The high percentage of frames detected as tonal (especially when using a short frame length, such as 32 samples) might seem slightly surprising, since the database contains sounds of a variety of birds (it was not specifically designed to contain tonal bird sounds only). This is contributed to by the fact that the use of such short frame length provides so coarse frequency resolution that even a non-tonal but frequency-localised signal would appear as tonal in the spectrum and thus would be detected. However, a coarse frequency resolution causes that a wider frequency region of noise can negatively affects the detection in noisy data. Let us now examine the performance on noisy data. We can see that the frame length of 128 samples provides the lowest detection performance in all noisy conditions. Comparing the results for the frame length of 32 and 64 samples as the SNR decreases, we can see that the frame length of 32 samples provides better detection 

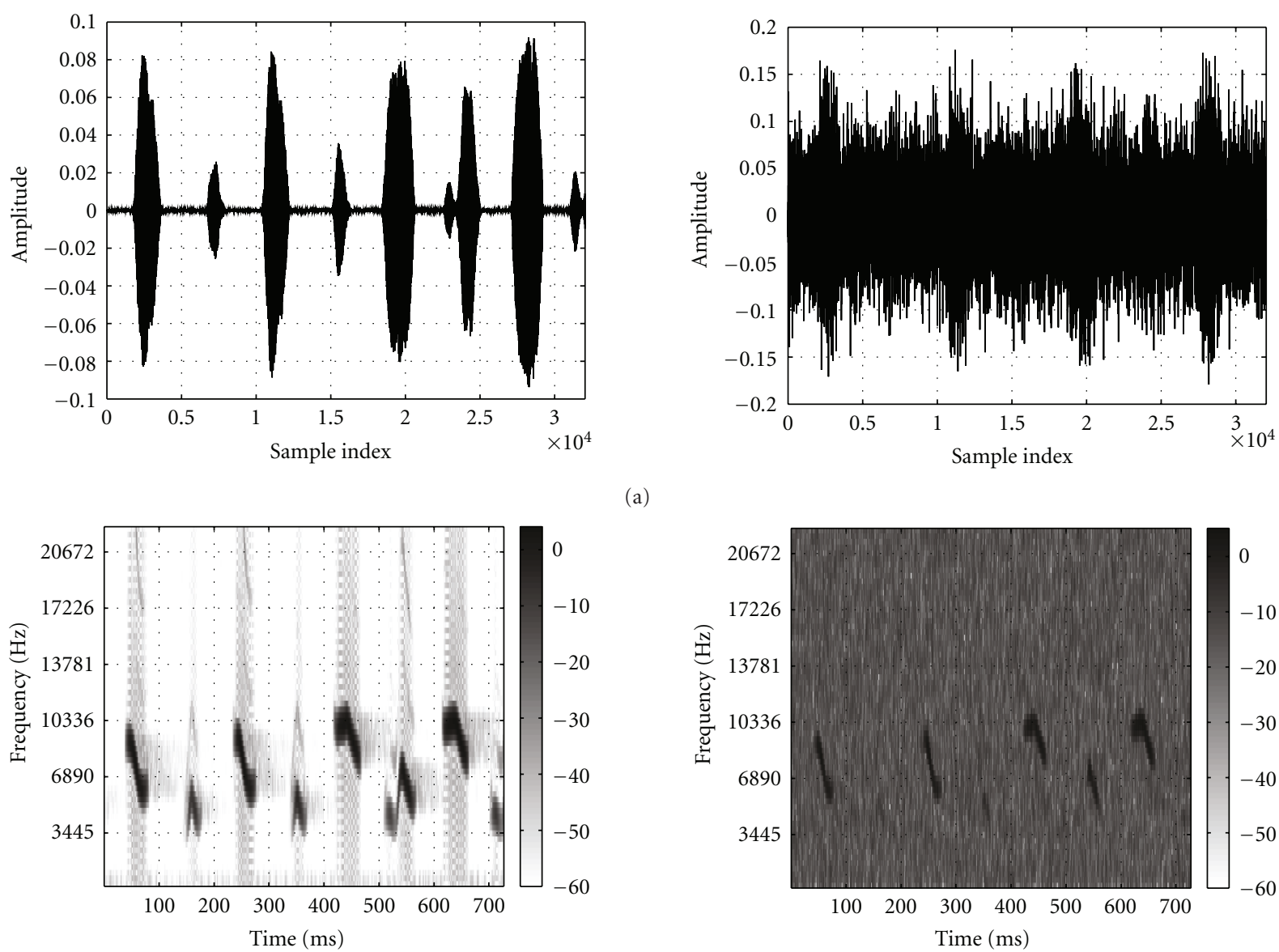

(b)
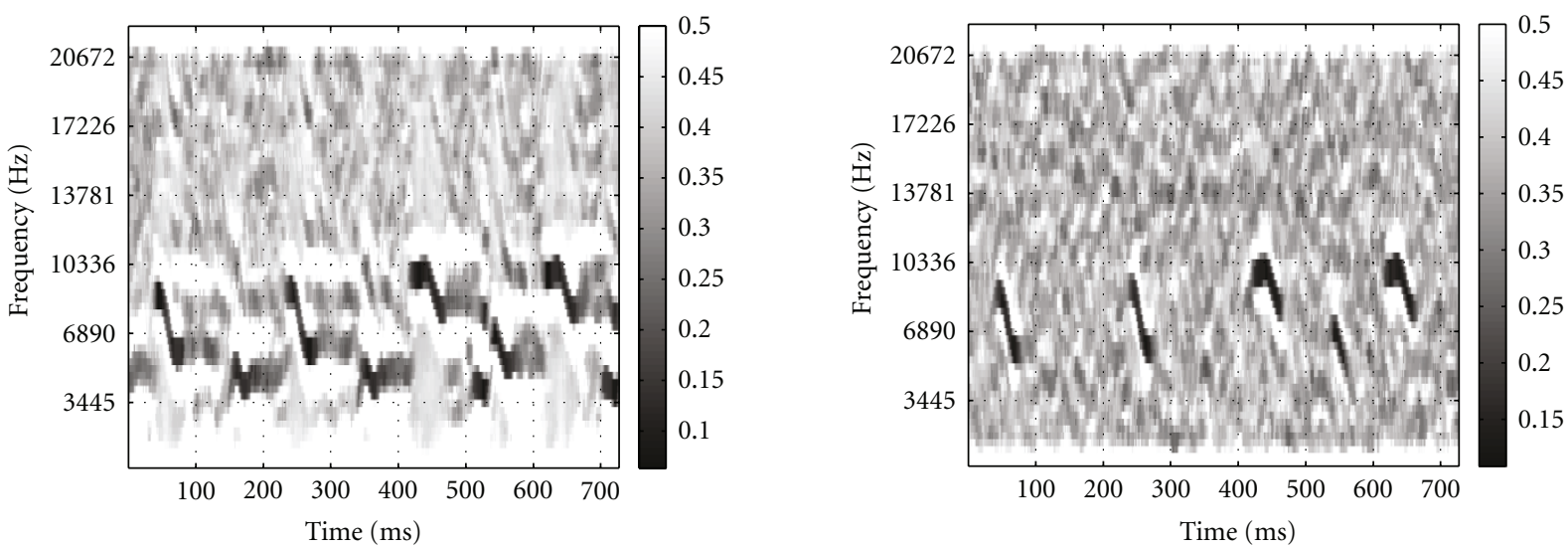

(c)

Figure 1: Waveform (a), spectrogram (b), and the corresponding sine-distance values (c) of a tonal bird song which is clean (left) and corrupted by White noise at the global SNR of $-10 \mathrm{~dB}$ (right).

performance at higher SNRs, while the frame length of 64 samples obtains better performance at lower SNRs. Since our main interest is the detection and recognition in noisy conditions and since the 32 samples frame length provides a very coarse frequency resolution, the frame length of 64 samples is used for the remaining experiments presented in this paper.
Let us now discuss the choice of tonal-threshold. The results presented in Figure 2 show that by increasing the value of the tonal-threshold, the amount of detected bird signal frames increases, but so does the false-acceptance error exponentially. For instance, in the case of global SNR of $-10 \mathrm{~dB}$, the increase of the bird signal frames detection from $36.5 \%$ to $54.7 \%$, which is around 1.5 times, would 


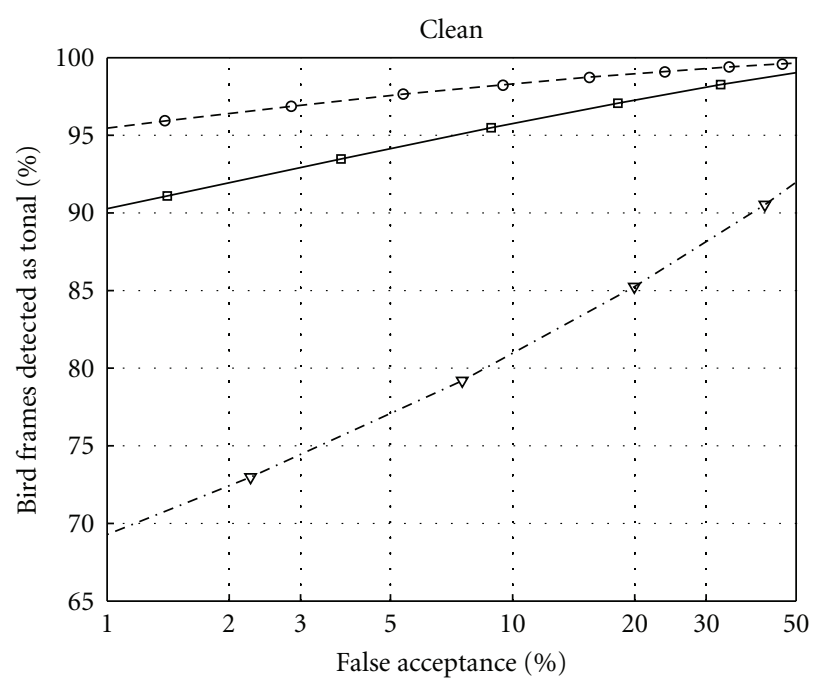

(a)

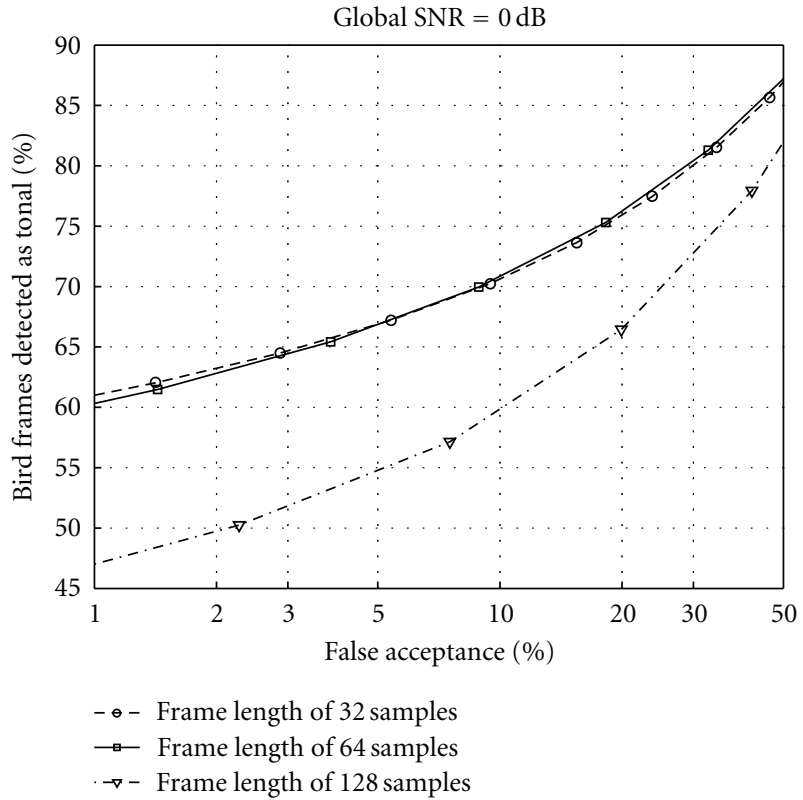

(c)

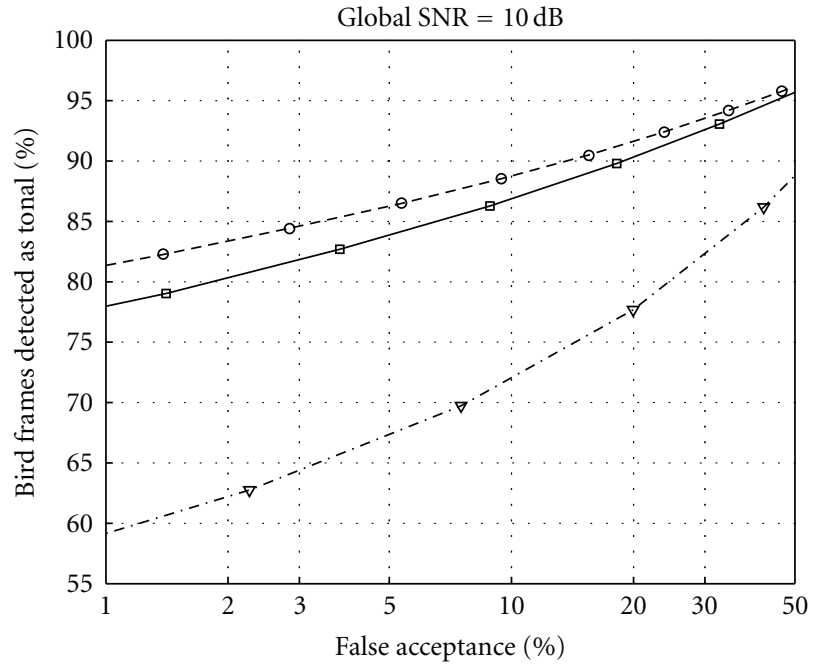

(b)

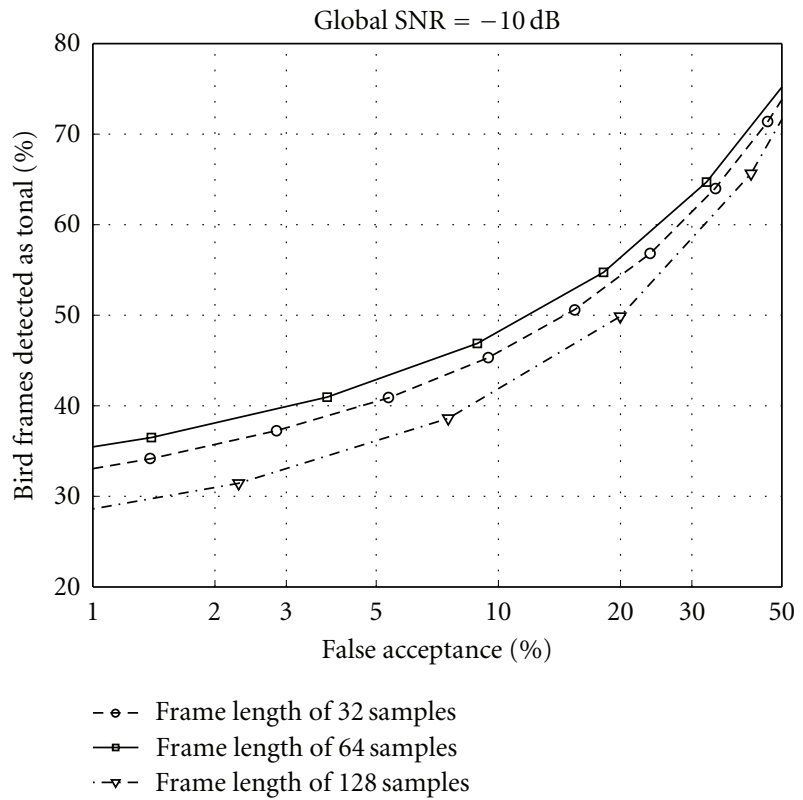

(d)

FIGURE 2: Percentage of frames detected as tonal on bird data ( $y$-axis) versus on White noise ( $x$-axis; referred to as false-acceptance). Bird data: clean (a) and corrupted by White noise at various global SNRs (b)-(d). Frame length [samples]: 32 (circle dashed line), 64 (square full line), and 128 (triangle dash-dotted line).

cause the false-acceptance error to increase 13 times from $1.4 \%$ to $18.2 \%$. Including large amount of falsely detected frames in recognition may have a more negative effect on the recognition performance than the reduced number of bird frames detected as tonal. We decided to choose a tonalthreshold which would result in a small false-acceptance error. Thus, the tonal-threshold was set to 0.24 , giving a $1.4 \%$ frame false-acceptance error.

Next, we will analyse the detection performance in terms of how many bird species are detected as having tonal singing in the database. This is performed for the frame length set to 64 samples and the tonal-threshold set to 0.24 , which gave $1.4 \%$ false-acceptance error at the frame-level. The results presented in Figure 3 depict the number of birds ( $y$-axis) having the given percentage of detected bird signal frames as tonal ( $x$-axis). The results show that 96 out of 99 birds had over $73 \%$ of the signal frames detected as tonal and no bird had less than $45 \%$ of the frames detected as tonal. This demonstrates that the proposed detection method may be applicable for detection of a large number of bird species.

Finally, we performed an evaluation of the detection of bird tonal regions at the spectral-level as a function of the local SNR. The local SNR for a given frequency point was calculated as the ratio of the energy of the clean signal and energy of the noise, each energy obtained as the average over energies at three frequency points around the 


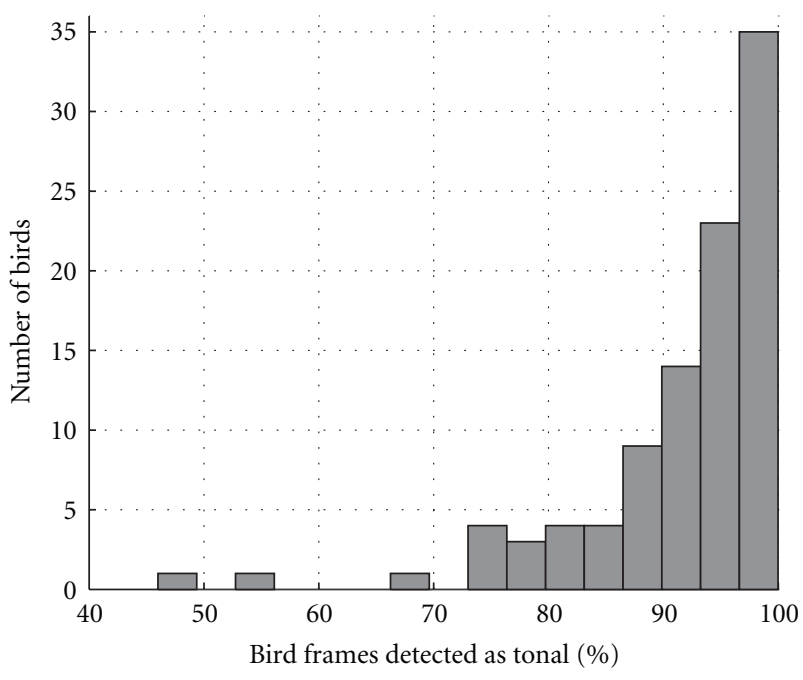

FIgURE 3: Histogram of the number of birds having the given percentage of bird signal frames detected as tonal on clean data.

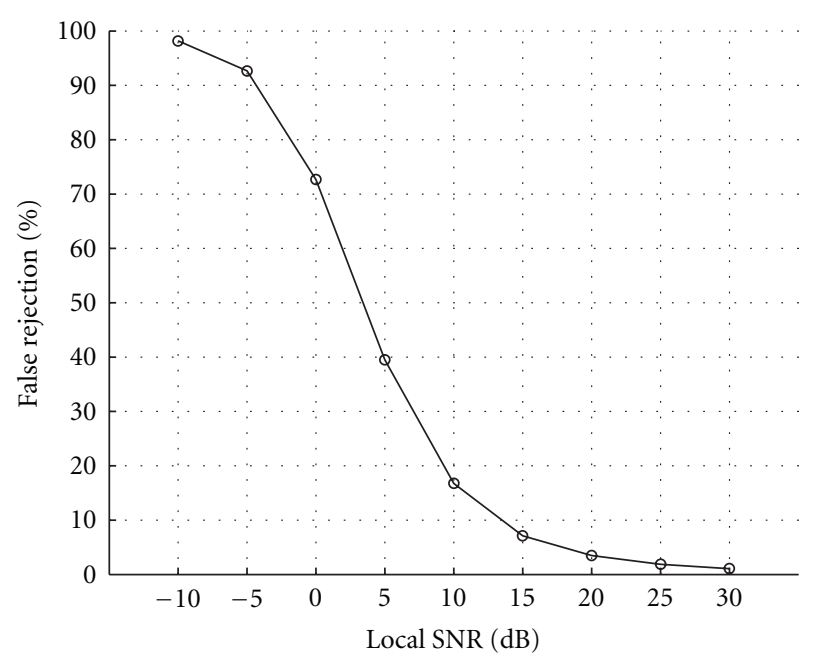

FIGURE 4: False-rejection error rate of bird tonal spectral points detection in White noise conditions as a function of the local SNR when the false-acceptance error was kept at $0.046 \%$.

considered frequency point. The signal frames detected as tonal on clean bird data were collected across all the noisy bird data corrupted at various global SNRs and used for this evaluation. The tonal-threshold was set to 0.24 , which resulted in $0.046 \%$ false-acceptance error at the spectrallevel, that is, the percentage of spectral points which were not detected as tonal on clean data but were detected as tonal on noisy data. The experimental results in terms of the falserejection error as a function of the local SNR are depicted in Figure 4. The false-rejection error refers to the percentage of spectral points which were detected as tonal on clean bird data but not detected on the noisy bird data at a given local SNR. We can see that even at the local SNR of $0 \mathrm{~dB}$, which corresponds to the energy of the signal and noise being equal, the false rejection is around $72 \%$, that is, approximately $28 \%$ of the bird tonal spectral points are still correctly detected.

\section{Automatic Bird Recognition}

This section presents our research on the employment of the spectral-level detection information provided by the method described in Section 2 for the recognition of bird syllables in noisy environments. The recognition system consists of two main parts: feature representation and modelling of the features. The following subsections describe first the probabilistic modelling of bird features and then the bird signal feature representations we employed. These are followed by experimental evaluations.

3.1. Probabilistic Modelling. The bird recognition system we employed is based on modelling the distribution of acoustic feature vectors for each bird syllable using the Gaussian mixture model (GMM). We employed GMMs as they were shown to achieve the best bird recognition performance in recent study in [8].

An $L$-component GMM $\lambda$ is a linear combination of $L$ Gaussian probability density functions and has the form

$$
p(\mathbf{y} \mid \lambda)=\sum_{l=1}^{L} w_{l} b_{l}(\mathbf{y})
$$

where $\mathbf{y}$ denotes the feature vector, $w_{l}$ is the weight and $b_{l}(\mathbf{y})$ is the density of the lth mixture component. The mixture weights satisfy the constraint $\sum_{l=1}^{L} w_{l}=1$. Each $b_{l}(\mathbf{y})$ is a multivariate Gaussian density of the form

$$
b_{l}(\mathbf{y})=\frac{1}{(2 \pi)^{D / 2}\left|\Sigma_{l}\right|^{1 / 2}} \exp \left(-\frac{1}{2}\left(\mathbf{x}-\mu_{l}\right)^{\prime} \Sigma_{l}^{-1}\left(\mathbf{x}-\mu_{l}\right)\right)
$$

with the mean vector $\mu_{l}$ and covariance matrix $\Sigma_{l}$. Gaussian densities with diagonal covariance matrix were used in this paper. Each bird syllable $s$ is represented by a GMM denoted by $\lambda_{s}$ which consists of the mixture weights and the mean vectors and covariance matrices of the Gaussian mixture components, that is, $\lambda_{s}=\left\{w_{l}, \mu_{l}, \Sigma_{l}\right\}_{l=1}^{L}$.

In recognition, we are given a sequence of feature vectors $Y=\left\{\mathbf{y}_{1}, \ldots, \mathbf{y}_{T}\right\}$, where $T$ is the number of frames. The objective of the recognition is to find the bird model $\lambda_{s}$ which gives the maximum a-posteriori probability for the given observation sequence $Y$, that is,

$$
s^{*}=\arg \max _{s} P\left(\lambda_{s} \mid Y\right) \propto \arg \max _{s} P\left(\lambda_{s}\right) p\left(Y \mid \lambda_{s}\right),
$$

where $s^{*}$ denotes the index of the bird syllable model achieving the maximum a-posteriori probability and $P\left(\lambda_{s}\right)$ is the a-priori probability of the bird syllable $s$, which we consider here to be equal for all bird syllables. Assuming independence between the observations and using the logarithm, the bird syllable recognition can then be written as

$$
s^{*}=\arg \max _{s} \sum_{t=1}^{T} \log p\left(\mathbf{y}_{t} \mid \lambda_{s}\right),
$$

where the $p\left(\mathbf{y}_{t} \mid \lambda_{s}\right)$ is calculated using (2) and (3). 
3.2. Feature Representation. The purpose of feature representation is to convert the signal into a sequence of feature vectors $Y$ that represent the information of interest in the signal. Our aim is to investigate an employment of tonalbased features which are obtained using the spectral-level detection method presented in Section 2. Since the previous research in automatic bird recognition has shown that the Mel-frequency cepstral coefficients (MFCC), which are currently the most widely used features for speech/speaker recognition, achieved the best performance for bird recognition, for example, [8], we used the MFCC features for comparison. The following subsections describe both types of feature representations. Both feature representations were obtained by dividing the signal into frames of 64 samples, with an overlap of 32 samples between frames and Hamming window was applied to each frame.

3.2.1. Mel-Frequency Cepstral Coefficients. The MFCC features were obtained as follows. The short-time magnitude spectrum, obtained by applying the FFT on each windowed signal frame, was passed to Mel-spaced filter-bank analysis. The obtained logarithm filter-bank energies were transformed using the discrete cosine transform, and the lower coefficients formed the static MFCC feature vector. In order to include dynamic spectral information, the first-order delta features, calculated as in [21] using two frames before and after the current frame, were added to the static MFCC feature vector.

In order to find the best parameter setup for the MFCC features, we performed experiments on clean data with the number of filter-bank (FB) channels set to a value from 10 to 50 and for each case the number of the cepstral coefficients set to 8,12 , and 20. Little differences in recognition accuracy were observed-the MFCC features used in all of the following experiments were obtained using $30 \mathrm{FB}$ channels and taking the first 20 cepstral coefficients. The addition of the delta features resulted in 40 dimensional MFCC feature vector for each signal frame.

3.2.2. Tonal-Based Features. The tonal-based features were obtained based on the tonal spectral detection method presented in Section 2. The static tonal-based feature vector for a given frame comprised of the frequency value and the logarithm of the magnitude value of the most prominent tonal component detected over the entire frequency range, that is, in a case a bird sound consisted of several frequency components (e.g., harmonics), only the information about the largest magnitude frequency component was used. The delta features capturing the dynamic information, calculated as mentioned in the previous section, were added to the static features, resulting in a 4 dimensional tonal-based feature vector (as opposed to the 40 dimensional in the case of MFCC).

\subsection{Experimental Evaluation of Bird Syllable Recognition}

3.3.1. Data Description and Experimental Setup. The database used for experiments was described earlier in
Section 2.3.1. The entire data, containing songs and calls of 99 birds, were manually split into individual syllable groups, each group consisting of a set of syllables with a similar spectral content, giving 281 different bird syllable groups. The data of each bird syllable was split (as detailed below) into a separate training set and testing set, which were then used for estimating the parameters of the GMMs and the experimental evaluations, respectively. Experiments were performed by employing both the standard models and noise-compensated models. The standard models were trained using the clean training data. The noise-compensated models were obtained by using multi-condition training approach, that is, the models were trained using a set of noisy training data. The training and testing data were obtained as follows. For each bird syllable, the detection of bird tonal frames was performed as described in Section 2 on clean data, and two thirds of the detected frames were allocated as the clean training data set. For each noisy conditions, the noisy training data set then consisted of the signal frames detected as tonal on the noise-corrupted versions of the training data. The clean and noisy testing sets consisted then of all the detected signal frames which did not belong to the training data. Note that the testing data included also the signal frames which were detected as tonal due to false-acceptance. In order to have a reasonable amount of the training data to train the models, only those bird syllables which had at least 250 frames detected as tonal on clean and noisy training data sets were used for the recognition experiments - this resulted in 165 out of 281 different bird syllables which were used for recognition experiments in this section. The experiments were performed with noisy bird data created by adding noise to the original data at global SNRs from $-10 \mathrm{~dB}$ to $10 \mathrm{~dB}$, in $5 \mathrm{~dB}$ steps, respectively. In addition to using White noise, we also used a real-world Waterfall noise recorded in a forest environment with a waterfall [20].

3.3.2. Experimental Results on the Standard Models. First, the evaluation of the proposed tonal-based features against the MFCC features was performed using standard models trained on clean data.

Recognition results obtained by the standard models using the MFCC and tonal-based features in clean conditions as a function of varying the number of mixture components in the model are presented in Table 1. It can be seen that using 16 and 32 mixture components provides the best performance for both types of features.

Next, experimental results obtained by the standard models using 32 mixture components for White and Waterfall noisy data are presented in Table 2. It can be seen that the MFCC features provide extremely low recognition performance even in mild noisy conditions at the SNR of $10 \mathrm{~dB}$. The failure of the MFCC features is due to capturing information from the entire spectrum, which may be largely dominated by noise since the bird sounds are often localised only in narrow frequency regions. On the other hand, the tonal-based features still provide very good performance even in strong noisy conditions at the SNR of $-10 \mathrm{~dB}$. 
TABLE 1: Bird syllable recognition accuracy on clean data obtained by the standard model having various number of mixture components and employing the MFCC and tonal-based features.

\begin{tabular}{lccccccc}
\hline Features & \multicolumn{7}{c}{ Number of mixture components } \\
\hline & 2 & 4 & 8 & 16 & 32 & 64 & 128 \\
\hline MFCC & 93.9 & 96.9 & 98.7 & 99.3 & 99.3 & 97.5 & 94.5 \\
Tonal & 60.6 & 75.1 & 88.4 & 95.7 & 95.7 & 92.1 & 87.8 \\
\hline
\end{tabular}

TABLE 2: Bird syllable recognition accuracy on noisy data obtained by the standard model employing the MFCC and tonal-based features.

\begin{tabular}{|c|c|c|c|c|c|c|c|c|c|c|}
\hline \multirow[t]{3}{*}{ Features } & \multicolumn{10}{|c|}{ Noisy conditions at a given SNR [dB] } \\
\hline & \multicolumn{5}{|c|}{ White noise } & \multicolumn{5}{|c|}{ Waterfall noise } \\
\hline & -10 & -5 & 0 & 5 & 10 & -10 & -5 & 0 & 5 & 10 \\
\hline MFCC & 0.6 & 0.6 & 1.2 & 3.0 & 9.7 & 0.6 & 0.6 & 1.2 & 2.4 & 9.0 \\
\hline Tonal & 50.3 & 61.8 & 72.7 & 83.6 & 86.0 & 56.9 & 67.2 & 78.1 & 83.6 & 87.8 \\
\hline
\end{tabular}

\subsubsection{Experimental Results on the Noise-Compensated Models.} In this section, we present the experimental results obtained by using noise-compensated models. These models were obtained by using the multi-condition training approach, which is often used in automatic speech recognition, for example, $[16,22]$.

First, results are presented for multi-condition models which were trained using the training data corrupted (at various SNR levels) by the same noise as used during the testing. This corresponds to real-world situations when the noise characteristics could be known a-priori or accurately estimated, for instance, when the noise is stationary as in the presence of a waterfall in the environment. Experimental evaluations showed in all cases that using 64 mixture components provided better performance than using 32 mixtures (used in the standard model). This reflects the increased variety of the training data. The obtained recognition results are presented in Table 3. It can be seen that the performance obtained by both the MFCC and tonal-based features when using the noise-compensated models is improved significantly in comparison to the results obtained by the standard model as in Table 2. Using the noise-compensated models, the tonal-based features provide significantly better performance than the MFCC features in most of the noisy conditions.

In a typical real-world scenario, environmental conditions vary, and it may not be possible to estimate noise characteristics reliably. In order to reflect this, we performed experiments where the training is based on an available noise, such as White noise, but the recognition is performed on a type of noise that was not seen during the training stage (in our case Waterfall noise). The results are presented in Figure 5. It can be seen that the recognition performance when using the MFCC features drops significantly in comparison to the previous case of matched training and testing noise conditions. As such, the MFCC features are not robust to the mismatch between training and testing noisy conditions. The proposed tonal-based features obtained recognition accuracy that is very close to the accuracy obtained when using the matched training and testing noisy conditions.

\section{Discussion and Conclusions}

Since bird sounds are often concentrated in a narrow frequency area, and in real-world conditions, there are often several birds singing simultaneously, the decomposition of the entire acoustic scene into individual sinusoidal components and their recombination at the classification stage seems a natural approach to take for detection and recognition of tonal bird sounds. In this paper, we presented a study of the detection and recognition of tonal bird sounds in noisy environments which follows this line of thought. We introduced a method for the detection of spectro-temporal regions of tonal birds sounds and then employed this for bird sound representation in a bird syllable recognition system. Experimental evaluations were performed on bird data from [19], which were corrupted by White noise and real-world Waterfall noise at various signal-to-noise ratios (SNRs).

The method we employed for bird sound detection exploits the principle of detecting sinusoidal components in the short-time spectrum based on spectral shape. It was shown that very short frame lengths, specifically 32 samples and 64 samples which correspond to $0.725 \mathrm{~ms}$ and $1.45 \mathrm{~ms}$, respectively, provided the best detection performance. This reflects the presence of fast frequency variations in bird sounds. The use of such short frame lengths is in contrast to previous works on automatic bird recognition, which often used the frame length from 5.8 to $11.6 \mathrm{~ms}$, for example, $[6,8]$. The use of such longer frame lengths would provide better frequency resolution, but, due to the fast frequency variations in bird sounds, it would also lead to some smearing in the spectrum. This has not been a problem for previous studies since they were not concerned with the detection of sinusoidal components, but only with a framelevel feature extraction.

The proposed detection method, when used at the framelevel, showed that over $95 \%$ of the clean bird signal frames in the bird database we used can be detected as tonal with false-acceptance of only $1 \%$. As such, this method can be used to provide an accurate automatic segmentation of a recorded signal into individual syllables. In previous 
TABLE 3: Bird syllable recognition accuracy on noisy data obtained by the multi-condition model employing the MFCC and tonal-based features.

\begin{tabular}{lcccccccccc}
\hline Features & \multicolumn{1}{c}{ Noisy conditions at a given SNR [dB] } \\
\hline & -10 & -5 & 0 & 5 & 10 & -10 & -5 & 0 & 5 & 10 \\
\hline MFCC & 54.5 & 75.7 & 86.6 & 92.7 & 95.1 & 50.3 & 79.3 & 84.8 & 93.9 & 97.5 \\
Tonal & 70.9 & 84.2 & 91.5 & 92.7 & 95.7 & 69.7 & 85.4 & 94.5 & 96.3 & 95.1 \\
\hline
\end{tabular}

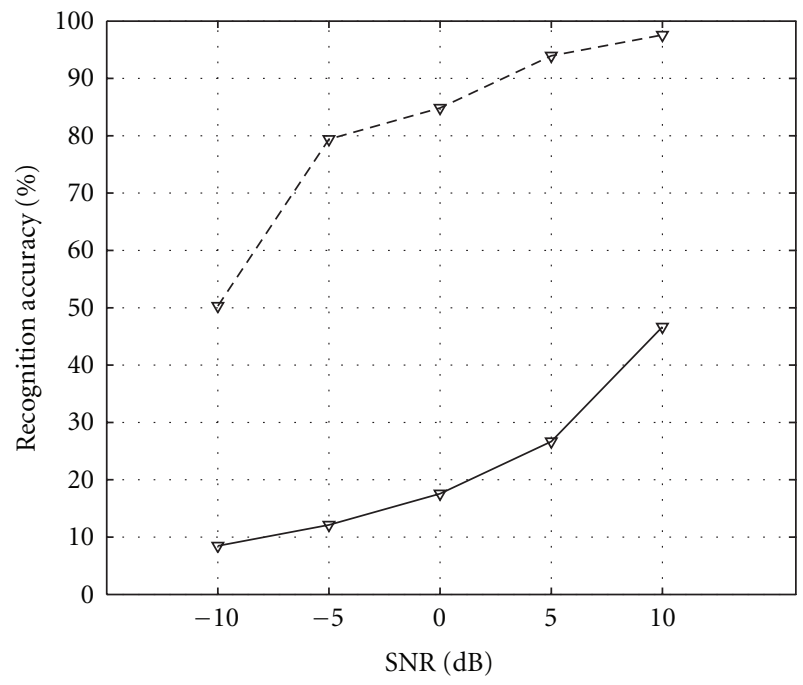

$\rightarrow$ MFCC (train-test mismatch)

$-\nabla-$ MFCC (train-test match)

(a)

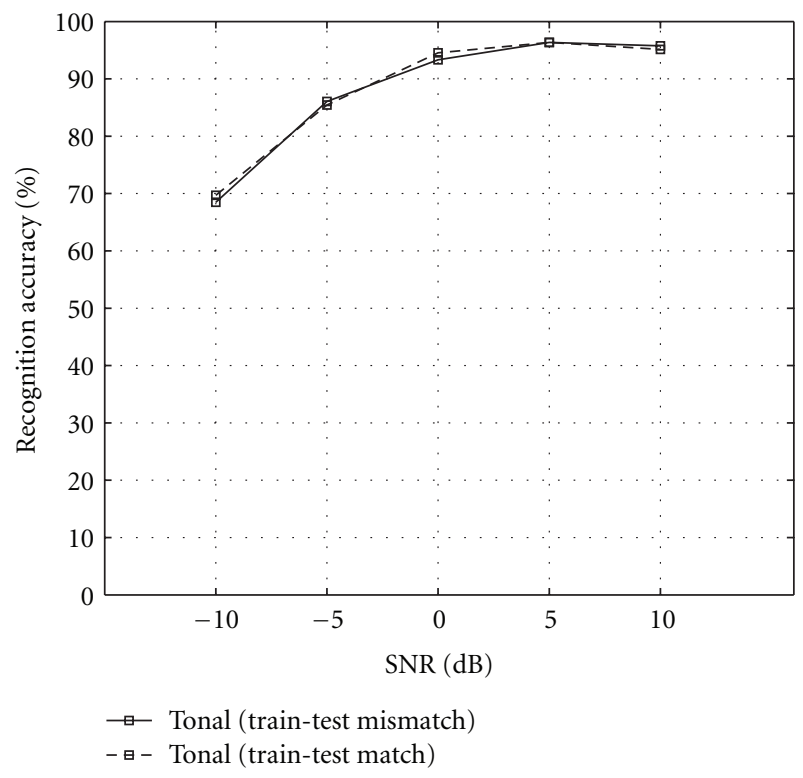

(b)

Figure 5: Bird syllable recognition accuracy on data corrupted by Waterfall noise obtained by the multi-condition model trained on Waterfall noise (train-test match) and White noise (train-test mismatch) and employing the MFCC (a) and the tonal-based (b) features. studies, for example, $[8,9]$, the syllable segmentation was performed based on a threshold defined by an estimate of the background noise energy level. This may be difficult to estimate accurately in non-stationary noisy environments with sudden noise and varying levels of noise.

The choice of the detection threshold, termed as tonalthreshold, determines the tradeoff between the correct detection rate and false-acceptance error rate. We set the tonalthreshold so as to achieve a very low false-acceptance error, since falsely detected regions may be seriously detrimental to the recognition accuracy. It was demonstrated that the proposed method provides very high accuracy in detecting the bird tonal spectral components in noisy environments. For instance, at $10 \mathrm{~dB}$ local SNR, the correct detection of bird tonal spectral components was around $83 \%$ while the falseacceptance was kept at only $0.046 \%$.

In the second part of the paper, we explored the representation of bird signals formed based on the output of the proposed tonal detection method. Specifically, the frequency and amplitude of the detected sinusoidal components were used, and these were referred to as tonal-based features. The work in [8] employed similar features, however, they were obtained based on the sinusoidal modelling algorithm presented in [13] and actually corresponded to the highest peak in the spectrum. The authors reported that the recognition performance obtained by these features was inferior to the conventional MFCC features. Moreover, the use of the highest peak in the spectrum would not be robust to noise, since a peak corresponding to any strong noise present in a different frequency region would be found instead of the peak corresponding to bird sound. The tonal-based features we employed in the study here showed very high recognition performance even in very strong noisy conditions. It was also shown that the performance can be further improved by using models trained on noise-corrupted training data, since such models can accommodate the effect of noise. The use of the same noise conditions for training the models, and testing is generally impossible in real-world scenario. When there was a mismatch between the training and testing noisy conditions, the currently most widely used MFCC features achieved very low recognition accuracy, while the proposed tonal-based features showed nearly the same performance as in the case of matched training-testing conditions.

In real-world scenario, there are usually several birds singing simultaneously. The proposed detection method can be directly employed for this scenario, since it provides the information on individual detected sinusoidal components for each signal frame. The recognition of birds singing 
simultaneously could then be performed by employing a multiple-hypothesis recognition approach. This is part of our future research work.

\section{Acknowledgment}

This work was partly supported by UK EPSRC Grant EP/ F036132/1.

\section{References}

[1] N. H. Fletcher, "A class of chaotic bird calls?" Journal of the Acoustical Society of America, vol. 108, no. 2, pp. 821-826, 2000.

[2] S. E. Anderson, A. S. Dave, and D. Margoliash, "Templatebased automatic recognition of birdsong syllables from continuous recordings," Journal of the Acoustical Society of America, vol. 100, pp. 1209-1219, 1996.

[3] J. A. Kogan and D. Margoliash, "Automated recognition of bird song elements from continuous recordings using dynamic time warping and hidden Markov models: a comparative study," Journal of the Acoustical Society of America, vol. 103, no. 4, pp. 2185-2196, 1998.

[4] A. L. Mcllraith and H. C. Card, "Birdsong recognition using backpropagation and multivariate statistics," IEEE Transactions on Signal Processing, vol. 45, no. 11, pp. 2740-2748, 1997.

[5] S. A. Selouani, M. Kardouchi, E. Hervet, and D. Roy, "Automatic birdsong recognition based on autoregressive timedelay neural networks," in Proceedings of the Congress on Computational Intelligence Methods and Applications (ICSC '05), pp. 1-6, Istanbul, Turkey, December 2005.

[6] C. F. Juang and T. M. Chen, "Birdsong recognition using prediction-based recurrent neural fuzzy networks," Neurocomputing, vol. 71, no. 1-3, pp. 121-130, 2007.

[7] C. Kwan, K. C. Ho, G. Mei et al., "An automated acoustic system to monitor and classify birds," EURASIP Journal on Applied Signal Processing, vol. 2006, Article ID 96706, 19 pages, 2006.

[8] P. Somervuo, A. Härmä, and S. Fagerlund, "Parametric representations of bird sounds for automatic species recognition," IEEE Transactions on Audio, Speech and Language Processing, vol. 14, no. 6, pp. 2252-2263, 2006.

[9] S. Fagerlund, "Bird species recognition using support vector machines," EURASIP Journal on Advances in Signal Processing, vol. 2007, Article ID 38637, 8 pages, 2007.

[10] A. Selin, J. Turunen, and J. T. Tanttu, "Wavelets in recognition of bird sounds," EURASIP Journal on Advances in Signal Processing, vol. 2007, Article ID 51806, 9 pages, 2007.

[11] C. Lee, Y. Lee, and R. Huang, "Automatic recognition of bird songs using cepstral coefficients," Journal of Information Technology and Applications, vol. 1, no. 1, pp. 17-23, 2006.

[12] A. Franzen and I. Y. H. Gu, "Classification of bird species by using key song searching: a comparative study," in Proceedings of the IEEE International Conference on Systems, Man and Cybernetics, vol. 1, pp. 880-887, October 2003.

[13] E. Bryan George and M. J. T. Smith, "Speech analysis/synthesis and modification using an analysis-by-synthesis/overlap-add sinusoidal model," IEEE Transactions on Speech and Audio Processing, vol. 5, no. 5, pp. 389-406, 1997.
[14] A. Harma, "Automatic recognition of bird species based on sinusoidal modeling of syllables," in Proceedings of the IEEE International Conference on Acoustics, Speech, and Signal Processing, pp. 545-548, Hong-Kong, China, 2003.

[15] P. Jančovič and M. Köküer, "Estimation of voicing-character of speech spectra based on spectral shape," IEEE Signal Processing Letters, vol. 14, no. 1, pp. 66-69, 2007.

[16] P. Jančovič and M. Köküer, "Incorporating the voicing information into HMM-based automatic speech recognition in noisy environments," Speech Communication, vol. 51, no. 5, pp. 438-451, 2009.

[17] P. Jančovič and M. Köküer, "Employment of spectral voicing information for speech and speaker recognition in noisy conditions," in Speech Recognition (Technologies and Applications), chapter 3, pp. 45-60, InTech, 2008.

[18] P. Jančovič and M. Köküer, "Improving automatic phoneme alignment under noisy conditions by incorporating spectral voicing information," Electronics Letters, vol. 45, no. 14, pp. 761-762, 2009.

[19] L. Elliott, Stokes Field Guide to Bird Songs: Eastern Region, 2009.

[20] "Waterfall noise," downloaded from http://www.freesound .org, a copy also available at http://www.eee.bham.ac.uk/ jancovic/research/Data.htm.

[21] S. Young, D. Kershaw, J. Odell, D. Ollason, V. Valtchev, and P. Woodland, The HTK Book. V2.2, 1999.

[22] H. Hirsch and D. Pearce, "The AURORA experimental framework for the performance evaluations of speech recognition systems under noisy conditions," in Proceedings of the International Symposium on Computer Architecture and International Tutorial and Research Workshop (ISCA ITRW ASR '00), pp. 181-188, Challenges for the New Millenium, Paris, France, September 2000. 